\title{
The Stochastic Coupling of SLE on the Strip Domain
}

\author{
Shenghua Zou \\ The Department of Basic Course Education, Ji'an College, Ji'an, China \\ Email: zoushenghua111@foxmail.com
}

How to cite this paper: Zou, S.H. (2020) The Stochastic Coupling of SLE on the Strip Domain. Journal of Applied Mathematics and Physics, 8, 1896-1910.

https://doi.org/10.4236/jamp.2020.89143

Received: August 21, 2020

Accepted: September 19, 2020

Published: September 22, 2020

Copyright $\odot 2020$ by author(s) and Scientific Research Publishing Inc. This work is licensed under the Creative Commons Attribution International License (CC BY 4.0).

http://creativecommons.org/licenses/by/4.0/

\begin{abstract}
The main work of this paper is to discuss the stochastic coupling of stirp $\operatorname{SLE}_{\kappa}(\kappa \in(0,4])$ on the stirp region $\mathbb{S}_{\pi}$. By constructing a bounded continuous local martingale, we prove that when a certain ordinary differential equation is satisfied, there is a coupling of two strip $\mathrm{SLE}_{\kappa}$ traces in $\mathbb{S}_{\pi}$; one is from $a$ to $b$; the other is from $b$ to $a$, such that the two curves visit the same set of points.
\end{abstract}

Keywords

Strip $\operatorname{SLE}_{\kappa}, \operatorname{SLE}_{\kappa}$ Trace, Stochastic Coupling

\section{Introduction}

Stochastic Loewner evolution (SLE) is a family of random growth process introduced by Oded Schramm [1] to study the scaling limit of loop-erased random walk (LERW) and uniform spanning tree (UST). The family of random growth process is described by the classic Loewner differential equation driven by $\sqrt{\kappa} B(t)$, where $\kappa$ is a positive parameter, and $B(t)$ is a one-dimensional standard Brownian motion. The behavior of SLE trace depends on the real-valued parameter $\kappa>0$; usually we write SLE as $\operatorname{SLE}_{\kappa}$ to illustrate that the behavior of SLE traces is related to $\kappa$. When $\kappa \in(0,4]$, the trace of $\operatorname{SLE}_{\kappa}$ is a simple curve; when $\kappa>4$, the trace is no longer a simple curve; when $\kappa>8$, the trace fills the whole space.

SLE is an important and very cutting-edge research topic in today's mathematics field, which involves random processes, complex analysis and statistical physics. It is closely related to the scale limit of the grid model in statistical physics. Many mathematicians believe that different $\kappa$ describes the scale lim- 
its of different discrete models. In statistical physics, the scale limits of many two-dimensional systems are conjectured by theoretical physicists to be conformal invariant under critical conditions, but it has not been not proven by rigorous mathematical methods. Since Oded Schramm introduced $\mathrm{SLE}_{\kappa}$, a lot of conjectures have been proven, see [2]-[8].

The stochastic coupling technique is a useful tool in studying reversibility of stochastic Loewner evolution (SLE). Dapeng Zhan proved the coupling of the chordal SLE in the process of proving the reversibility of the chordal SLE in [9]. He then proved the coupling of the annulus SLE and the whole-plane SLE in [10], and on this basis he proved that the whole-plane SLE is reversible, which is closely related with Julien Dub's work on SLE couple relationships in [11] [12]. The stochastic couplings of strip SLE has not been studied so far. The research of this paper will lay the foundation for the study of stirp SLE reversibility.

This paper is organized as follows. In Section 2, we give some symbols that will be used frequently in this paper. The definition of strip $\operatorname{SLE}(\kappa, \Lambda)$ is introduced in Section 3. In Section 4, we construct a continuous local martingale $M$ based on (4) (5), and then prove that $M$ is bounded. On this basis, we prove that for $\kappa \in(0,4]$, there is a coupling of two strip $\operatorname{SLE}_{\kappa}$ process on the strip domain.

\section{Symbols}

In this article, we will use the following symbols: Let $\mathbb{S}_{\pi}=\{z \in \mathbb{C}: 0<\operatorname{Im} z<\pi\}$, $\mathbb{R}_{\pi}=\{z \in \mathbb{C}: \operatorname{Im} z=\pi\}, \overline{\mathbb{D}}=\{z:|z|<1\}, \mathbb{T}=\{z:|z|=1\}$. The conformal map in this paper refers to a univalent analytic function. Let $f$ be the conformal in $D_{1}$, and $f\left(D_{1}\right)=D_{2}, f$ is said to be conformal map from $D_{1}$ onto $D_{2}$, denoted as $f: D_{1} \rightarrow D_{2}$. Further, if $j=1,2, c_{j}$ is points or collections in $\partial D_{j}$, and $f$ extension map $c_{1}$ onto $c_{2}$, denoted as $f:\left(D_{1} ; c_{1}\right) \stackrel{\text { conf }}{\rightarrow}\left(D_{2} ; c_{2}\right)$.

Many of the functions in this text have two variables, the first of which represent time, and the second is not. In this case, We use $\partial_{t}$ and $\partial_{t}^{n}$ to represent the partial derivative of the first variable, and $d_{j}(j=1,2)$ is used to represent the differentials of $t_{j}$. We'll use $\operatorname{coth}(z / 2)$ frequently. For convenience, we will write 2 in the position of subscript, namely $\operatorname{coth}_{2}(z)=\operatorname{coth}(z / 2)$.

\section{Strip Loewner Equation}

In this section we give a brief description of the definition and some basic concepts of the strip Loewner equation, and more detailed background can be found in [13] [14].

Definition 3.1. Let $T \in(0,+\infty), \xi(t) \in C([0, T))$. Let $g(t, z)$ be the solution of

$$
\partial_{t} g(t, z)=\operatorname{coth}_{2}(g(t, z)-\xi(t)), g(t, z)=z .
$$

For each $t \in[0, T)$, let $K(t)$ be the set of $z \in \overline{\mathbb{S}}$ at which $g(t, z)$ is not defined. Then $K(t)$ and $g(t, z), 0 \leq t<T$ are called the strip Loewner hulls 
and maps driven by $\xi(t)$. For each $t \in[0, T), K(t)$ is a bounded random growth hull in $\overline{\mathbb{S}}_{\pi}$ and $\operatorname{dist}\left(K(t), \mathbb{R}_{\pi}\right)>0$, $g(t, \cdot):\left(\mathbb{S}_{\pi} \backslash K(t) ; \mathbb{R}_{\pi}\right) \stackrel{\text { conf }}{\rightarrow}\left(\mathbb{S}_{\pi} ; \mathbb{R}_{\pi}\right), \quad g(t, z)-z \rightarrow \pm t, \quad \mathbb{S}_{\pi} \backslash K(t) \ni z \rightarrow \pm \infty$.

Let $K$ is a bounded hull in $\mathbb{S}_{\pi}$, and $\operatorname{dist}\left(K, \mathbb{R}_{\pi}\right)>0$. Then there is a constant $c_{K} \geq 0$, and a map $g_{K}$ determined by $K$, such that $g_{K}:\left(\mathbb{S}_{\pi} \backslash K ; \mathbb{R}_{\pi}\right) \stackrel{\text { conf }}{\rightarrow}\left(\mathbb{S}_{\pi} ; \mathbb{R}_{\pi}\right), \quad g_{K}-z \rightarrow \pm c_{k}, \mathbb{S}_{\pi} \backslash K \ni z \rightarrow \pm \infty . \quad c_{K}$ is called the capacity of $K$ with respect to $\mathbb{R}_{\pi}$ in $\mathbb{S}_{\pi}$, denote $\operatorname{scap}(K(t))$. Then, for the above strip Loewner hulls, the capacity of $K(t)$ is $t$.

Let $\xi(t)$ is a semi-martingale, whose stochastic part is $\sqrt{\kappa} B(t)$ and drift part is a continuously differentiable function. Then

$$
\gamma(t):=\lim _{\mathbb{S}_{\pi} \ni z \rightarrow \xi(t)} g^{-1}(t, z), 0 \leq t<T,
$$

a.s. for any $t \in[0, T), \gamma(t)$ exists. It is a continuous curve in $\mathbb{S}_{\pi} \cup \mathbb{R}$, who starte from $\xi(0)$. We call $\gamma(t)$ the strip Loewner trace driven by $\xi(t)$. For each $t \in[0, T), \mathbb{S}_{\pi} \backslash K(t)$ is the unbounded branch of $\mathbb{S}_{\pi} \backslash \gamma((0, t])$. Particularly, when $0<\kappa \leq 4, \gamma(t)$ is a simple curves, for each $t \in[0, T)$, $K(t)=\gamma((0, t])$.

On the other hand, Let $\gamma(t)$ be a simple curves in $\mathbb{S}_{\pi} \cup \mathbb{R}$, and only intersecting with $\mathbb{R}$ when $t=0$. Let $u(t)$ be the capacity of $\gamma(t)$ with respect to $\mathbb{R}_{\pi}$ in $\mathbb{S}_{\pi}$. Then $u(t)$ is a continuous increase function, which maps $[0, T)$ to $[0, S)$ ( $S$ is a constant in $(0,+\infty))$. there exist some $\eta(t) \in C([0, S))$ so that $\gamma\left(u^{-1}(t)\right)$ is a strip Loewner trace driven by $\eta(t)$.

Definition 3.2. Let $a>b \in \mathbb{R}, \Lambda \in C^{1}((0,+\infty))$. Let $\xi(t), 0 \leq t<T$ be the maximal solution to the SDE:

$$
\mathrm{d} \xi(t)=\sqrt{\kappa} \mathrm{d} B(t)+\Lambda(\xi(t)-g(t, b)) \mathrm{d} t, \xi(0)=a,
$$

where $g(t, \cdot)$ is a strip Loewner maps driven by $\xi(t)$. We call the strip Loewner trace driven by $\xi(t)$ the strip $\operatorname{SLE}(\kappa, \Lambda)$ trace in $\mathbb{S}_{\pi}$ started from a with marked point $b$.

\section{Coupling of Two Strip SLE Trace}

In this chapter we will discuss the stochastic coupling of the traces of strip SLE. We prove that for $\kappa \in(0,4]$, when certain ODE is satisfied, we can couple two strip SLE trace. That is, we have the following theorem.

Theorem 4.1. Let $\kappa \in(0,4], s_{0} \in \mathbb{R}$, Suppose $\Gamma \in C^{2}((0,+\infty))$ is a positive function that satisfies

$$
\begin{aligned}
& 0=\frac{\kappa}{2} \Gamma^{\prime \prime}+\operatorname{coth}_{2} \Gamma^{\prime}+\left(\frac{3}{\kappa}-\frac{1}{2}\right) \operatorname{coth}_{2}^{\prime} \Gamma, \\
& \Gamma(x+2 \pi)=\mathrm{e}^{\frac{2 \pi s_{0}}{\kappa}} \Gamma(x), \quad x \in(0,+\infty) .
\end{aligned}
$$

Let $\Lambda=\kappa \frac{\Gamma^{\prime}}{\Gamma}, \Lambda_{1}(x)=\Lambda(x), \Lambda_{2}(x)=-\Lambda(-x)$, then $\forall a_{1}>a_{2} \in \mathbb{R}$, there is a coupling of two curves: $\gamma_{1}(t), 0 \leq t<T_{1}$ and $\gamma_{2}(t), 0 \leq t<T_{2}$, such that for $j \neq k \in\{1,2\}$, 
(i) $\gamma_{j}(t), 0 \leq t<T_{j}$ is the strip $\operatorname{SLE}(\kappa, \Lambda)$ trace in $\mathbb{S}_{\pi}$ started from $a_{j}$ with marked point $a_{k}$.

(ii) If $t_{k} \in\left[0, T_{k}\right)$ is a stopping time with respect to $\gamma_{k}(t)$, then conditioned on $\gamma_{k}(t), 0 \leq t \leq t_{k}$, After a time-change, $\gamma_{j}(t), 0 \leq t<T_{j}$, is the strip $\operatorname{SLE}\left(\kappa ; \Lambda_{j}\right)$ trace in $\mathbb{S}_{\pi} \backslash \beta_{k}\left(\left(0, t_{k}\right]\right)$ started from $a_{j}$ with marked point $\gamma_{k}\left(t_{k}\right)$, where $T_{j}\left(t_{k}\right)$ is the first time that $\gamma_{j}$ visits $\gamma_{k}\left(\left(0, t_{k}\right]\right)$, if such time not exist set to be $T_{j}$.

\subsection{Ensemble}

Let $T>0, \quad \xi_{1}, \xi_{2} \in C([0, T]), \quad g_{j}(t, \cdot)$ and $\gamma_{j}(t, \cdot)(j=1,2), 0 \leq t<T$ are the strip Loewner map and trace driven by $\xi(t)$. Define

$$
\mathcal{D}=\left\{\left(t_{1}, t_{2}\right): \beta_{1}\left(\left[0, t_{1}\right]\right) \cap \beta_{2}\left(\left[0, t_{2}\right]\right)=\varnothing\right\} .
$$

Fix $j \neq k \in 1,2, \quad t_{k} \in[0, T)$, let $T_{j}\left(t_{k}\right)$ is the first time that $\gamma_{j}$ visits $\gamma_{k}\left(\left(0, t_{k}\right]\right)$. Define

$$
\gamma_{j, t_{k}}\left(t_{j}\right)=g_{k}\left(t_{k}, \gamma_{j}\left(t_{j}\right)\right), \quad 0 \leq t_{j}<T_{j}\left(t_{k}\right) .
$$

Then $\gamma_{j, t_{k}}\left(t_{j}\right), 0 \leq t_{j}<T_{j}\left(t_{k}\right)$ is a simple curves start from $g_{k}\left(t_{k}, \xi_{j}(0)\right) \in \mathbb{R}$, when $0 \leq t_{j}<T_{j}\left(t_{k}\right), \gamma_{j, t_{k}}\left(\left(0, t_{j}\right]\right) \subseteq \mathbb{S}_{\pi}$. Let $u_{j, t_{k}}\left(t_{j}\right)=\operatorname{scap}\left(\gamma_{j, t_{k}}\left(\left(0, t_{j}\right]\right)\right)$, then $u_{j, t_{k}}$ is a continuous increase function, which maps $\left[0, T_{j}\left(t_{k}\right)\right)$ to $\left[0, S_{j, t_{k}}\right)$, where $S_{j, t_{k}}=\sup u_{j, t_{k}}\left[0, T_{j}\left(t_{k}\right)\right)$. $\beta_{j, t_{k}}(t):=\gamma_{j, t_{k}}\left(u_{j, t_{k}}^{-1}(t)\right), 0 \leq t \leq S_{j, t_{k}}$ is a strip Loewner trace driven by some $\eta_{j, t_{k}} \in C\left(\left[0, S_{j, t_{k}}\right)\right)$.

Let $f_{j, t_{k}}(t)$ be a strip Loewner trace map by $\eta_{j, t_{k}}$. For $0 \leq t_{j}<T_{j}\left(t_{k}\right)$, let $g_{j, t_{k}}\left(t_{j}, \cdot\right)=f_{j, t_{k}}\left(u_{j, t_{k}}\left(t_{j}, \cdot\right)\right), \quad \xi_{j, t_{k}}(t)=\eta_{j, t_{k}}\left(u_{j, t_{k}}(t)\right)$,

$$
G_{k, t_{k}}\left(t_{j}, \cdot\right)=g_{j, t_{k}}\left(t_{j}, \cdot\right) \circ g_{k}\left(t_{k}, \cdot\right) \circ g_{j}^{-1}\left(t_{j}, \cdot\right) .
$$

$$
\begin{aligned}
G_{k, t_{k}}\left(t_{j}, \cdot\right) \operatorname{map} & \mathbb{S}_{\pi} \backslash \beta_{k, t_{j}}\left(\left(0, t_{k}\right]\right) \text { to } \mathbb{S}_{\pi}, \operatorname{map} \mathbb{R}_{\pi} \text { to } \mathbb{R}_{\pi} . \\
\eta_{j, t_{k}}\left(t_{j}\right)= & f_{j, t_{k}}\left(t_{j}, \beta_{j, t_{k}}\left(t_{j}\right)\right) \\
= & f_{j, t_{k}}\left(t_{j}, \cdot\right) \circ \gamma_{j, t_{k}}\left(u_{j, t_{k}}^{-1}\left(t_{j}\right)\right) \\
& =f_{j, t_{k}}\left(t_{j}, \cdot\right) \circ g_{j, t_{k}}\left(t_{j}, \cdot\right) \circ \gamma_{j}\left(u_{j, t_{k}}^{-1}\left(t_{j}\right)\right) \\
& =f_{j, t_{k}}\left(t_{j}, \cdot\right) \circ g_{j, t_{k}}\left(t_{j}, \cdot\right) \circ g_{j}^{-1}\left(u_{j, t_{k}}\left(t_{j}\right), \cdot\right) \circ \xi_{j}\left(u_{j, t_{k}}^{-1}\left(t_{j}\right)\right),
\end{aligned}
$$

Hence,

$$
\xi_{j, t_{k}}\left(t_{j}\right)=G_{k, t_{k}}\left(t_{j}, \xi_{j}\left(t_{j}\right)\right)
$$

For $0 \leq t_{j}<T_{j}\left(t_{k}\right)$, let

$$
\begin{gathered}
A_{j, n}\left(t_{1}, t_{2}\right)=G_{k, t_{k}}^{(n)}\left(t_{j}, \xi\left(t_{j}\right)\right), \quad n=1,2,3, \\
A_{j, S}\left(t_{1}, t_{2}\right)=\frac{A_{j, 3}\left(t_{1}, t_{2}\right)}{A_{j, 1}\left(t_{1}, t_{2}\right)}-\frac{3}{2}\left(\frac{A_{j, 2}\left(t_{1}, t_{2}\right)}{A_{j, 1}\left(t_{1}, t_{2}\right)}\right)^{2} .
\end{gathered}
$$


By [15], Section 8.1

$$
u_{j, t_{k}}^{\prime}\left(t_{j}\right)=G_{k, t_{k}}^{\prime}\left(t, \xi_{j}\left(t_{j}\right)\right)^{2}=A_{j, 1}^{2}\left(t_{1}, t_{2}\right) .
$$

So for $0 \leq t_{j}<T_{j}\left(t_{k}\right)$,

$$
\begin{aligned}
\partial_{t} g_{j, t_{k}}\left(t_{j}, \cdot\right) & =\partial_{t} f_{j, t_{k}}\left(u_{j, t_{k}}\left(t_{j}\right), \cdot\right) u_{j, t_{k}}^{\prime}\left(t_{j}\right) \\
& =A_{j, 1}^{2}\left(t_{1}, t_{2}\right) \operatorname{coth}_{2}\left(g_{j, t_{k}}\left(t_{j}, \cdot\right)-\xi_{j, t_{k}}\left(t_{j}\right)\right) .
\end{aligned}
$$

From (6) we get

$$
G_{k, t_{k}}\left(t_{j}, \cdot\right) \circ g_{j}\left(t_{j}, z\right)=g_{j, t_{k}}\left(t_{j}, \cdot\right) \circ g_{k}\left(t_{k}, z\right) .
$$

Differentiate (12) with respect to $t_{j}$, we get

$$
\begin{aligned}
& \partial_{t}\left(g_{j, t_{k}}\left(t_{j}, \cdot\right) \circ g_{k}\left(t_{k}, z\right)\right) \\
& =\partial_{t} G_{k, t_{k}}\left(t_{j}, \cdot\right) \circ g_{j}\left(t_{j}, z\right)+G_{k, t_{k}}^{\prime}\left(t_{j}, \cdot\right) \circ g_{j}\left(t_{j}, z\right)\left(\operatorname{coth}_{2}\left(g_{j}\left(t_{j}, z\right)-\xi_{j}\left(t_{j}\right)\right)\right) \\
& =A_{j, 1}^{2}\left(t_{1}, t_{2}\right) \operatorname{coth}_{2}\left(g_{j, t_{k}}\left(t_{j}, \cdot\right) \circ g_{k}\left(t_{k}, z\right)-\xi_{j, t_{k}}\left(t_{j}\right)\right) \\
& =A_{j, 1}^{2}\left(t_{1}, t_{2}\right) \operatorname{coth}_{2}\left(G_{k, t_{k}}\left(t_{j}, \cdot\right) \circ g_{j}\left(t_{j}, z\right)-\xi_{j, t_{k}}\left(t_{j}\right)\right) .
\end{aligned}
$$

Let $\omega=g_{j}\left(t_{j}, z\right)$, then

$$
\begin{aligned}
& \partial_{t} G_{k, t_{k}}\left(t_{j}, \omega\right)+G_{k, t_{k}}^{\prime}\left(t_{j}, \omega\right) \operatorname{coth}_{2}\left(\omega-\xi_{j}\left(t_{j}\right)\right) \\
& =A_{j, 1}^{2}\left(t_{1}, t_{2}\right) \operatorname{coth}_{2}\left(G_{k, t_{k}}\left(t_{j}, \omega\right)-\xi_{j, t_{k}}\left(t_{j}\right)\right) .
\end{aligned}
$$

Hence,

$$
\begin{aligned}
\partial_{t} G_{k, t_{k}}\left(t_{j}, \omega\right)= & -G_{k, t_{k}}^{\prime}\left(t_{j}, \omega\right) \operatorname{coth}_{2}\left(\omega-\xi_{j}\left(t_{j}\right)\right) \\
& +A_{j, 1}^{2}\left(t_{1}, t_{2}\right) \operatorname{coth}_{2}\left(G_{k, t_{k}}\left(t_{j}, \omega\right)-\xi_{j, t_{k}}\left(t_{j}\right)\right) .
\end{aligned}
$$

The Taylor expansion of $\operatorname{coth}_{2} z, \operatorname{coth}_{2}^{\prime} z$ near $z \rightarrow 0$ is:

$$
\operatorname{coth}_{2} z=\frac{2}{z}+\frac{z}{6}+o\left(z^{2}\right), \operatorname{coth}_{2}^{\prime} z=-\frac{2}{z^{2}}+\frac{1}{6}+o(z) .
$$

Let $\omega \rightarrow \xi_{j}\left(t_{j}\right)$, from (7), (15) and L'Hopital's Rule,

$$
\partial_{t} G_{k, t_{k}}\left(t_{j}, \xi_{j}\left(t_{j}\right)\right)=-3 A_{j, 2}\left(t_{1}, t_{2}\right) .
$$

Differentiate (14) with respect to $\omega$. Let $\omega \rightarrow \xi_{j}\left(t_{j}\right)$, from (7), (15)and L'Hopital's Rule,

$$
\frac{\partial_{t} G_{k, t_{k}}^{\prime}\left(t_{j}, \xi_{j, t_{k}}\left(t_{j}\right)\right)}{G_{k, t_{k}}^{\prime}\left(t_{j}, \xi_{j, t_{k}}\left(t_{j}\right)\right)}=\frac{1}{6} A_{j, 1}^{2}\left(t_{1}, t_{2}\right)-\frac{1}{6}-\frac{4}{3} \frac{A_{j, 3}\left(t_{1}, t_{2}\right)}{A_{j, 1}\left(t_{1}, t_{2}\right)}+\frac{1}{2} \frac{A_{j, 2}^{2}\left(t_{1}, t_{2}\right)}{A_{j, 1}^{2}\left(t_{1}, t_{2}\right)} .
$$

$G_{k, t_{k}}\left(t_{j}, \cdot\right)$ and $g_{k, t_{j}}\left(t_{k}, \cdot\right)$ map $\mathbb{S}_{\pi} \backslash \beta_{k, t_{j}}\left(\left(0, t_{k}\right]\right)$ conformal onto $\mathbb{S}_{\pi}$, map $\mathbb{R}_{\pi}$ conformal onto $\mathbb{R}_{\pi}$. So exist $C_{k}\left(t_{1}, t_{2}\right) \in \mathbb{R}$, such that

$$
G_{k, t_{k}}\left(t_{j}, \cdot\right)=g_{k, t_{j}}\left(t_{k}, \cdot\right)+C_{k}\left(t_{1}, t_{2}\right) .
$$

Similarly exist $C_{j}\left(t_{1}, t_{2}\right) \in \mathbb{R}$, such that

$$
G_{j, t_{k}}\left(t_{j}, \cdot\right)=g_{j, t_{j}}\left(t_{k}, \cdot\right)+C_{j}\left(t_{1}, t_{2}\right) .
$$


From (6),

$$
g_{j, t_{k}}\left(t_{j}, \cdot\right) \circ g_{k}\left(t_{k}, \cdot\right)=g_{k, t_{j}}\left(t_{k}, \cdot\right) \circ g_{j}\left(t_{j}, \cdot\right)+C_{k}\left(t_{1}, t_{2}\right) .
$$

Similarly,

$$
g_{k, t_{j}}\left(t_{k}, \cdot\right) \circ g_{j}\left(t_{j}, \cdot\right)=g_{j, t_{k}}\left(t_{j}, \cdot\right) \circ g_{k}\left(t_{k}, \cdot\right)+C_{j}\left(t_{1}, t_{2}\right) .
$$

Comparing (20) with (21), we get

$$
C_{1}\left(t_{1}, t_{2}\right)+C_{2}\left(t_{1}, t_{2}\right) \equiv 0 .
$$

Define $X_{1}\left(t_{1}, t_{2}\right), X_{2}\left(t_{1}, t_{2}\right),\left(t_{1}, t_{2}\right) \in \mathcal{D}$ :

$$
\begin{aligned}
X_{j}\left(t_{1}, t_{2}\right) & =\xi_{j, t_{k}}\left(t_{j}\right)-g_{j, t_{k}}\left(t_{j}, \xi_{k}\left(t_{k}\right)\right) \\
& =G_{k, t_{k}}\left(t_{j}, \xi_{j}\left(t_{j}\right)\right)-g_{j, t_{k}}\left(t_{j}, \xi_{k}\left(t_{k}\right)\right) .
\end{aligned}
$$

From (18), (19), (23),

$$
X_{1}\left(t_{1}, t_{2}\right)+X_{2}\left(t_{1}, t_{2}\right) \equiv 0 \text {. }
$$

Since $\operatorname{coth}_{2}$ is an odd function, $\operatorname{coth}_{2}^{\prime \prime \prime}$ is an even. Define $E\left(t_{1}, t_{2}\right),\left(t_{1}, t_{2}\right) \in \mathcal{D}$

$$
E\left(t_{1}, t_{2}\right)=\operatorname{coth}_{2}^{\prime \prime \prime} X_{1}\left(t_{1}, t_{2}\right)=\operatorname{coth}_{2}^{\prime \prime \prime} X_{2}\left(t_{1}, t_{2}\right) .
$$

Differentiate (11) with respect to $Z$, we have

$$
\partial_{t} g_{j, t_{k}}^{\prime}\left(t_{j}, z\right)=A_{j, 1}^{2} g_{j, t_{k}}^{\prime}\left(t_{j}, z\right) \operatorname{coth}_{2}^{\prime}\left(g_{j, t_{k}}\left(t_{j}, z\right)-\xi_{j, t_{k}}\left(t_{j}\right)\right) \text {. }
$$

so

$$
\begin{gathered}
\frac{\partial_{t} g_{j, t_{k}}^{\prime}\left(t_{j}, z\right)}{g_{j, t_{k}}^{\prime}\left(t_{j}, z\right)}=A_{j, 1}^{2} \operatorname{coth}_{2}^{\prime}\left(g_{j, t_{k}}\left(t_{j}, z\right)-\xi_{j, t_{k}}\left(t_{j}\right)\right) . \\
\partial_{t} g_{j, t_{k}}^{\prime \prime}\left(t_{j}, z\right)=A_{j, 1}^{2} g_{j, t_{k}}^{\prime \prime}\left(t_{j}, z\right) \operatorname{coth}_{2}^{\prime}\left(g_{j, t_{k}}\left(t_{j}, z\right)-\xi_{j, t_{k}}\left(t_{j}\right)\right) \\
+A_{j, 1}^{2}\left(g_{j, t_{k}}^{\prime}\left(t_{j}, z\right)\right)^{2} \operatorname{coth}_{2}^{\prime \prime}\left(g_{j, t_{k}}\left(t_{j}, z\right)-\xi_{j, t_{k}}\left(t_{j}\right)\right) .
\end{gathered}
$$

From (26) and (28) we have

$$
\begin{aligned}
\partial_{t}\left(\frac{g_{j, t_{k}}^{\prime \prime}\left(t_{j}, z\right)}{g_{j, t_{k}}^{\prime}\left(t_{j}, z\right)}\right) & =\frac{\left(\partial_{t} g_{j, t_{k}}^{\prime \prime}\left(t_{j}, z\right)\right) g_{j, t_{k}}^{\prime}\left(t_{j}, z\right)-\left(\partial_{t} g_{j, t_{k}}^{\prime}\left(t_{j}, z\right)\right) g_{j, t_{k}}^{\prime \prime}\left(t_{j}, z\right)}{\left(g_{j, t_{k}}^{\prime}\left(t_{j}, z\right)\right)^{2}} \\
& =A_{j, 1}^{2} g_{j, t_{k}}^{\prime}\left(t_{j}, z\right) \operatorname{coth}_{2}^{\prime \prime}\left(g_{j, t_{k}}\left(t_{j}, z\right)-\xi_{j, t_{k}}\left(t_{j}\right)\right) .
\end{aligned}
$$

Differentiate (29) with respect to $z$, we have

$$
\begin{aligned}
& \partial_{t}\left(\frac{g_{j, t_{k}}^{\prime \prime \prime}\left(t_{j}, z\right)}{g_{j, t_{k}}^{\prime}\left(t_{j}, z\right)}-\left(\frac{g_{j, t_{k}}^{\prime \prime}\left(t_{j}, z\right)}{g_{j, t_{k}}^{\prime}\left(t_{j}, z\right)}\right)^{2}\right) \\
& =A_{j, 1}^{2}\left(g_{j, t_{k}}^{\prime}\left(t_{j}, z\right)\right)^{2} \operatorname{coth}_{2}^{\prime \prime \prime}\left(g_{j, t_{k}}\left(t_{j}, z\right)-\xi_{j, t_{k}}\left(t_{j}\right)\right) \\
& \quad+A_{j, 1}^{2} g_{j, t_{k}}^{\prime \prime}\left(t_{j}, z\right) \operatorname{coth}_{2}^{\prime \prime}\left(g_{j, t_{k}}\left(t_{j}, z\right)-\xi_{j, t_{k}}\left(t_{j}\right)\right) .
\end{aligned}
$$

Let $z=\xi_{k}\left(t_{k}\right)$ in (11), (27), (29), (30) we have

$$
\partial_{j} g_{j, t_{k}}\left(t_{j}, \xi_{k}\left(t_{k}\right)\right)=-A_{j, 1}^{2} \operatorname{coth}_{2}\left(X_{j}\right) \text {, }
$$




$$
\begin{gathered}
\frac{\partial_{j} A_{k, 1}}{A_{k, 1}}=A_{j, 1}^{2} \operatorname{coth}_{2}^{\prime}\left(X_{j}\right), \\
\partial_{j}\left(\frac{A_{k, 2}}{A_{k, 1}}\right)=-A_{j, 1}^{2} A_{k, 1} \operatorname{coth}_{2}^{\prime \prime}\left(X_{j}\right), \\
\partial_{j}\left(\frac{A_{k, 3}}{A_{k, 1}}-\left(\frac{A_{k, 2}}{A_{k, 1}}\right)^{2}\right)=-A_{j, 1}^{2}\left[A_{k, 1}^{2} \operatorname{coth}_{2}^{\prime \prime \prime} X_{j}-A_{k, 2} \operatorname{coth}_{2}^{\prime \prime}\left(X_{j}\right)\right] .
\end{gathered}
$$

From (34) we have

$$
\partial_{j} A_{k, S}=\partial_{j}\left(\frac{A_{k, 3}}{A_{k, 1}}-\frac{3}{2}\left(\frac{A_{k, 2}}{A_{k, 1}}\right)^{2}\right)=A_{j, 1}^{2} A_{k, 1}^{2} E .
$$

Define $H\left(t_{1}, t_{2}\right),\left(t_{1}, t_{2}\right) \in \mathcal{D}$

$$
H\left(t_{1}, t_{2}\right)=\exp \left(\int_{0}^{t_{2}} \int_{0}^{t_{1}} A_{1,1}^{2}\left(s_{1}, s_{2}\right) A_{2,1}^{2}\left(s_{1}, s_{2}\right) E\left(s_{1}, s_{2}\right) \mathrm{d} s_{1} \mathrm{~d} s_{2}\right) .
$$

As $g_{j, t_{k}}(0, \cdot)=f_{j, t_{k}}(0, \cdot)=i d$, when $t_{j}=0, A_{k, 1}=0, A_{k, 2}=0, A_{k, 3}=0$, so $A_{k, S}=0$. Hence from (35), (36) we have

$$
\frac{\partial_{j} H}{H}=\partial_{j}(\ln H)=\partial_{j}\left(\int_{0}^{t_{2}} \int_{0}^{t_{1}} A_{1,1}^{2}\left(s_{1}, s_{2}\right) A_{2,1}^{2}\left(s_{1}, s_{2}\right) E\left(s_{1}, s_{2}\right) \mathrm{d} s_{1} \mathrm{~d} s_{2}\right)=A_{j, s} .
$$

\subsection{Transformations of ODE}

Lemma 4.2. If positive function $\Gamma \in C^{2}([0,+\infty))$ satisfy (4), $\Lambda:=\kappa \frac{\Gamma^{\prime}}{\Gamma}$, then

$$
0=\frac{\kappa}{2} \Lambda^{\prime \prime}+\left(3-\frac{\kappa}{2}\right) \operatorname{coth}_{2}^{\prime \prime}+\Lambda \operatorname{coth}_{2}^{\prime}+\operatorname{coth}_{2} \Lambda^{\prime}+\Lambda \Lambda .
$$

Proof.

$$
\Lambda:=\kappa \frac{\Gamma^{\prime}}{\Gamma},
$$

so

$$
(\ln \Gamma(s))^{\prime}=\frac{\Gamma(s)^{\prime}}{\Gamma(s)}=\frac{1}{\kappa} \Lambda(s) .
$$

Integral on both sides, we have

$$
\int_{1}^{x}(\ln \Gamma(s))^{\prime} \mathrm{d} s=\int_{1}^{x} \frac{1}{\kappa} \Lambda(s) \mathrm{d} s .
$$

i.e.

$$
\ln \Gamma(x)-\ln \Gamma(1)=\frac{1}{\kappa} \int_{1}^{x} \Lambda(s) \mathrm{d} s .
$$

so

$$
\Gamma(x)=\Gamma(1) \mathrm{e}^{\frac{1}{\kappa} \int_{1}^{x} \Lambda(s) \mathrm{d} s} .
$$

Thus 


$$
\begin{gathered}
\Gamma^{\prime}(x)=\frac{1}{\kappa} \Gamma(1) \mathrm{e}^{\frac{1}{\kappa} \int_{1}^{x} \Lambda(s) \mathrm{ds}} \Lambda(x), \\
\Gamma^{\prime \prime}(x)=\frac{1}{\kappa} \Gamma(1) \mathrm{e}^{\frac{1}{\kappa} \int_{1}^{x} \Lambda(s) \mathrm{d} s} \Lambda(x)^{2}+\frac{1}{\kappa} \Gamma(1) \mathrm{e}^{\frac{1}{\kappa} \int_{1}^{x} \Lambda(s) \mathrm{ds}} \Lambda^{\prime}(x) .
\end{gathered}
$$

From (4), we get

$$
\begin{aligned}
0 & =\frac{\kappa}{2} \Gamma^{\prime \prime}+\operatorname{coth}_{2} \Gamma^{\prime}+\left(\frac{3}{\kappa}-\frac{1}{2}\right) \operatorname{coth}_{2} \Gamma \\
& =\frac{1}{\kappa} \Gamma(1) \mathrm{e}^{\frac{1}{\kappa} \int_{1}^{x} \Lambda(s) \mathrm{ds}}\left[\frac{1}{2} \Lambda^{2}+\frac{\kappa}{2} \Lambda^{\prime}+\Lambda \operatorname{coth}_{2}+\left(3-\frac{\kappa}{2}\right) \operatorname{coth}_{2}^{\prime}\right] .
\end{aligned}
$$

Hence,

$$
\frac{1}{2} \Lambda^{2}+\frac{\kappa}{2} \Lambda^{\prime}+\Lambda \operatorname{coth}_{2}+\left(3-\frac{\kappa}{2}\right) \operatorname{coth}_{2}^{\prime}=0
$$

Differentiate with respect to $x$, we have

$$
\frac{\kappa}{2} \Lambda^{\prime \prime}+\left(3-\frac{\kappa}{2}\right) \operatorname{coth}_{2}^{\prime \prime}+\Lambda \operatorname{coth}_{2}^{\prime}+\operatorname{coth}_{2} \Lambda^{\prime}+\Lambda \Lambda^{\prime}=0
$$

\subsection{Martingales in Two Time Variables}

Let $a_{1}, a_{2}, \Gamma, \Lambda_{1}, \Lambda_{2}$ be as Theoerm 4.1. Let $B_{1}(t), B_{2}(t)$ be two independent Brownian motion, $\mathcal{F}_{t}^{j}=\sigma\left(B_{j}(s) ; 0 \leq s \leq t\right)(j=1,2)$. For $j=1,2$, Let $\xi_{j}\left(t_{j}\right), 0 \leq t_{j}<T_{j}$, be the solution of

$$
\mathrm{d} \xi_{j}\left(t_{j}\right)=\sqrt{\kappa} \mathrm{d} B_{j}\left(t_{j}\right)+\Lambda_{j}\left(\xi_{j}\left(t_{j}\right)-g_{j}\left(t_{j}, b\right)\right) \mathrm{d} t_{j}, \xi_{j}(0)=a_{j},
$$

then $\left(\xi_{1}\right),\left(\xi_{2}\right)$ are independent. When $\kappa \in(0,4], \xi_{j}\left(t_{j}\right)(j=1,2)$ is a.s. a simple curves, denoted by $\gamma_{j}\left(t_{j}\right) \cdot \gamma_{j}\left(t_{j}\right), g_{j}\left(t_{j}, \cdot\right)$ are driven by $\xi_{j}\left(t_{j}\right)$, Thus, They are $\mathcal{F}_{t_{j}}^{j}$-adapted. $\gamma_{j}\left(t_{j}\right)$ is $\mathcal{F}_{t_{j}}^{j}$-adapted, $g_{k}\left(t_{k}, \cdot\right)$ is $\mathcal{F}_{t_{k}}^{k}$-adapted, so $\left(t_{1}, t_{2}\right) \mapsto \gamma_{j, t_{k}}\left(t_{j}\right)=g_{k}\left(t_{k}, \beta_{j}\left(t_{j}\right)\right),\left(t_{1}, t_{2}\right) \in \mathcal{D}$ are $\left(\mathcal{F}_{t_{1}}^{1} \times \mathcal{F}_{t_{2}}^{2}\right)$-adapted. $f_{j, t_{k}}\left(t_{j}, \cdot\right)$ is determined by $\gamma_{j, t_{k}}\left(s_{j}\right), 0 \leq s_{j}<t_{j}$, hence, $f_{j, t_{k}}\left(t_{j}, \cdot\right)$ is $\left(\mathcal{F}_{t_{1}}^{1} \times \mathcal{F}_{t_{2}}^{2}\right)$-adapted. From (6) we get, $\left(G_{k, t_{k}}\left(t_{j}, \cdot\right)\right)$ is $\left(\mathcal{F}_{t_{1}}^{1} \times \mathcal{F}_{t_{2}}^{2}\right)$-adapted. From (7) we have, $\left(\xi_{j, t_{k}}\left(t_{j}\right)\right)$ is $\left(\mathcal{F}_{t_{1}}^{1} \times \mathcal{F}_{t_{2}}^{2}\right)$-adapted. From (8), (9), (23) we get, $\left(\xi_{j}\right),\left(A_{j, n}\right)(n=1,2,3)$ and $\left(A_{j, S}\right)$ are $\left(\mathcal{F}_{t_{1}}^{1} \times \mathcal{F}_{t_{2}}^{2}\right)$-adapted.

Fix $j \neq k \in\{1,2\}$ and a $\mathcal{F}_{t}^{k}$-stopping time $t_{k} \in\left[0, T_{k}\right)$. Let $\mathcal{F}_{t_{j}}^{j, t_{k}}=\mathcal{F}_{t_{j}}^{j} \times \mathcal{F}_{t_{k}}^{k}, 0 \leq t_{j}<T_{j}$, then $\left(\mathcal{F}_{t_{j}}^{j, t_{k}}\right)$ is a filtration. $B_{j}\left(t_{j}\right)$ is independent of $\mathcal{F}_{t_{k}}^{k}$, so it is a $\left(\mathcal{F}_{t_{j}}^{j, t_{k}}\right)$-Brownian motion. Hence, (39) is $\left(\mathcal{F}_{t_{j}}^{j, t_{k}}\right)$-adapted SDE.

From (23), (16), (8), (11), we get

$$
\begin{aligned}
\mathrm{d}_{j} X_{j} & =\mathrm{d}_{j} G_{k, t_{k}}\left(t_{j}, \xi_{j}\left(t_{j}\right)\right)-\mathrm{d}_{j} g_{j, t_{k}}\left(t_{j}, \xi_{k}\left(t_{k}\right)\right) \\
& =A_{j, 1} \mathrm{~d} \xi_{j}\left(t_{j}\right)+\left(\frac{\kappa}{2}-3\right) A_{j, 2} \mathrm{~d} t_{j}+A_{j, 1}^{2} \operatorname{coth}_{2} X_{j} \mathrm{~d} t_{j} .
\end{aligned}
$$

Let $\Gamma_{1}(x)=\Gamma(x), \quad \Gamma_{2}(x)=\Gamma(-x), \quad \Lambda_{j}=\kappa \frac{\Gamma_{j}^{\prime}}{\Gamma_{j}}(j=1,2)$. Suppose $\Gamma_{j}$ satisfy (4). From (24), we define $Y\left(t_{1}, t_{2}\right),\left(t_{1}, t_{2}\right) \in \mathcal{D}$ : 


$$
Y=\Gamma_{1}\left(X_{1}\right)=\Gamma_{2}\left(X_{2}\right) .
$$

From Itô formula and (4), (40) we have

$$
\begin{aligned}
\frac{\mathrm{d}_{j} Y}{Y}= & \frac{\Gamma_{j}^{\prime}\left(X_{j}\right) \mathrm{d}_{j} X_{j}+\frac{1}{2} \Gamma_{j}^{\prime \prime}\left(X_{j}\right)\left(\mathrm{d}_{j} X_{j}\right)^{2}}{\Gamma_{j}\left(X_{j}\right)} \\
= & \frac{1}{\kappa} \Lambda_{j}\left(X_{j}\right) A_{j, 1} \mathrm{~d} \xi_{j}\left(t_{j}\right)+\frac{1}{\kappa}\left(\frac{\kappa}{2}-3\right) A_{j, 2} \Lambda_{j}\left(X_{j}\right) \mathrm{d} t_{j} \\
& -\left(\frac{3}{\kappa}-\frac{1}{2}\right) A_{j, 1}^{2} \operatorname{coth}_{2}^{\prime}\left(X_{j}\right) \mathrm{d} t_{j} \\
= & \frac{1}{\kappa} \Lambda_{j}\left(X_{j}\right) A_{j, 1} \mathrm{~d} \xi_{j}\left(t_{j}\right)-\left(\frac{3}{\kappa}-\frac{1}{2}\right)\left(A_{j, 2} \Lambda_{j}\left(X_{j}\right)+A_{j, 1}^{2} \operatorname{coth}_{2}^{\prime}\left(X_{j}\right)\right) \mathrm{d} t_{j} .
\end{aligned}
$$

From Itô formula and (17) we have

$$
\begin{aligned}
\frac{\mathrm{d}_{j} A_{j, 1}}{A_{j, 1}} & =\frac{\mathrm{d} G_{k, t_{k}}^{\prime}\left(t_{j}, \xi_{j}\left(t_{j}\right)\right)}{A_{j, 1}} \\
& =\frac{A_{j, 2}}{A_{j, 1}} \mathrm{~d} \xi_{j}\left(t_{j}\right)+\left[\left(\frac{\kappa}{2}-\frac{4}{3}\right) \frac{A_{j, 3}}{A_{j, 1}}+\frac{1}{2}\left(\frac{A_{j, 2}}{A_{j, 1}}\right)^{2}+\frac{1}{6} A_{j, 1}^{2}-\frac{1}{6}\right] \mathrm{d} t_{j} .
\end{aligned}
$$

Let

$$
\alpha=\frac{6-\kappa}{2 \kappa}, \quad c=\frac{(8-3 \kappa)(\kappa-6)}{2 \kappa} .
$$

From Itô formula and (43), we get

$$
\begin{aligned}
\frac{\mathrm{d}_{j} A_{j, 1}^{\alpha}}{A_{j, 1}^{\alpha}} & =\frac{\alpha A_{j, 1}^{\alpha-1} \mathrm{~d}_{j} A_{j, 1}+\frac{1}{2} \alpha(\alpha-1) A_{j, 1}^{\alpha-2}\left(\mathrm{~d}_{j} A_{j, 1}\right)^{2}}{A_{j, 1}^{\alpha}} \\
& =\alpha \frac{\mathrm{d}_{j} A_{j, 1}}{A_{j, 1}}+\frac{1}{2} \alpha(\alpha-1)\left(\frac{\mathrm{d}_{j} A_{j, 1}}{A_{j, 1}}\right)^{2} \\
& =\alpha \frac{A_{j, 2}}{A_{j, 1}} \mathrm{~d} \xi_{j}\left(t_{j}\right)+\left(\frac{c}{6} A_{j, S}+\frac{1}{6} \alpha A_{j, 1}^{2}-\frac{1}{6} \alpha\right) \mathrm{d} t_{j} .
\end{aligned}
$$

From (19) and (32) we get

$$
\frac{\mathrm{d}_{j} A_{k, 1}}{A_{k, 1}}=\frac{\mathrm{d}_{j} G_{j, t_{j}}^{\prime}\left(t_{k}, \xi_{k}\left(t_{k}\right)\right)}{A_{k, 1}}=\frac{\partial_{j} A_{k, 1}}{A_{k, 1}}=A_{j, 1}^{2} \operatorname{coth}_{2}^{\prime}\left(X_{j}\right) \mathrm{d} t_{j} .
$$

Thus

$$
\frac{\mathrm{d}_{j} A_{k, 1}^{\alpha}}{A_{k, 1}^{\alpha}}=\alpha A_{j, 1}^{2} \operatorname{coth}_{2}^{\prime}\left(X_{j}\right) \mathrm{d} t_{j} .
$$

Define $\hat{M}\left(t_{1}, t_{2}\right),\left(t_{1}, t_{2}\right) \in \mathcal{D}$ :

$$
\hat{M}\left(t_{1}, t_{2}\right)=A_{1,1}^{\alpha} A_{2,1}^{\alpha} H^{-\frac{c}{6}} Y .
$$

Lemma 4.3. Let $X_{1}, X_{2}, X_{3}, X_{4}$ be a Itô process in $\mathbb{R}$, let $N_{t}=X_{1} X_{2} X_{3} X_{4}$, then 


$$
\begin{aligned}
\frac{\mathrm{d} N_{t}}{N_{t}}= & \frac{\mathrm{d} X_{1}}{X_{1}}+\frac{\mathrm{d} X_{2}}{X_{2}}+\frac{\mathrm{d} X_{3}}{X_{3}}+\frac{\mathrm{d} X_{4}}{X_{4}}+\frac{\mathrm{d} X_{1}}{X_{1}} \cdot \frac{\mathrm{d} X_{2}}{X_{2}}+\frac{\mathrm{d} X_{1}}{X_{1}} \cdot \frac{\mathrm{d} X_{3}}{X_{3}} \\
& +\frac{\mathrm{d} X_{1}}{X_{1}} \cdot \frac{\mathrm{d} X_{4}}{X_{4}}+\frac{\mathrm{d} X_{2}}{X_{2}} \cdot \frac{\mathrm{d} X_{3}}{X_{3}}+\frac{\mathrm{d} X_{2}}{X_{2}} \cdot \frac{\mathrm{d} X_{4}}{X_{4}}+\frac{\mathrm{d} X_{3}}{X_{3}} \cdot \frac{\mathrm{d} X_{4}}{X_{4}} .
\end{aligned}
$$

Proof. Process from Itô formula we have

$$
\mathrm{d}\left(X_{1} X_{2}\right)=X_{1} \mathrm{~d} X_{2}+X_{2} \mathrm{~d} X_{1}+\mathrm{d} X_{1} \cdot \mathrm{d} X_{2} .
$$

Then

$$
\frac{\mathrm{d}\left(X_{1} X_{2}\right)}{X_{1} X_{2}}=\frac{\mathrm{d} X_{1}}{X_{1}}+\frac{\mathrm{d} X_{2}}{X_{2}}+\frac{\mathrm{d} X_{1}}{X_{1}} \cdot \frac{\mathrm{d} X_{2}}{X_{2}}
$$

Thus

$$
\begin{aligned}
\frac{\mathrm{d}\left(X_{1} X_{2} X_{3}\right)}{X_{1} X_{2} X_{3}} & =\frac{X_{1} X_{2} \mathrm{~d} X_{3}+X_{3} \mathrm{~d}\left(X_{1} X_{2}\right)+\mathrm{d}\left(X_{1} X_{2}\right) \cdot \mathrm{d} X_{3}}{X_{1} X_{2} X_{3}} \\
& =\frac{\mathrm{d} X_{3}}{X_{3}}+\frac{\mathrm{d}\left(X_{1} X_{2}\right)}{X_{1} X_{2}}+\frac{\mathrm{d}\left(X_{1} X_{2}\right)}{X_{1} X_{2}} \cdot \frac{\mathrm{d} X_{3}}{X_{3}} \\
& =\frac{\mathrm{d} X_{1}}{X_{1}}+\frac{\mathrm{d} X_{2}}{X_{2}}+\frac{\mathrm{d} X_{3}}{X_{3}}+\frac{\mathrm{d} X_{1}}{X_{1}} \cdot \frac{\mathrm{d} X_{2}}{X_{2}}+\frac{\mathrm{d} X_{1}}{X_{1}} \cdot \frac{\mathrm{d} X_{3}}{X_{3}}+\frac{\mathrm{d} X_{2}}{X_{2}} \cdot \frac{\mathrm{d} X_{3}}{X_{3}} .
\end{aligned}
$$

and

$$
\begin{aligned}
\frac{\mathrm{d}\left(X_{1} X_{2} X_{3} X_{4}\right)}{X_{1} X_{2} X_{3} X_{4}}= & \frac{X_{1} X_{2} X_{3} \mathrm{~d} X_{4}+X_{4} \mathrm{~d}\left(X_{1} X_{2} X_{3}\right)+\mathrm{d}\left(X_{1} X_{2} X_{3}\right) \cdot \mathrm{d} X_{4}}{X_{1} X_{2} X_{3} X_{4}} \\
= & \frac{\mathrm{d} X_{4}}{X_{4}}+\frac{\mathrm{d}\left(X_{1} X_{2} X_{3}\right)}{X_{1} X_{2} X_{3}}+\frac{\mathrm{d}\left(X_{1} X_{2} X_{3}\right)}{X_{1} X_{2} X_{3}} \cdot \frac{\mathrm{d} X_{4}}{X_{4}} \\
= & \frac{\mathrm{d} X_{1}}{X_{1}}+\frac{\mathrm{d} X_{2}}{X_{2}}+\frac{\mathrm{d} X_{3}}{X_{3}}+\frac{\mathrm{d} X_{4}}{X_{4}}+\frac{\mathrm{d} X_{1}}{X_{1}} \cdot \frac{\mathrm{d} X_{2}}{X_{2}}+\frac{\mathrm{d} X_{1}}{X_{1}} \cdot \frac{\mathrm{d} X_{3}}{X_{3}} \\
& +\frac{\mathrm{d} X_{2}}{X_{2}} \cdot \frac{\mathrm{d} X_{3}}{X_{3}}+\frac{\mathrm{d} X_{1}}{X_{1}} \cdot \frac{\mathrm{d} X_{4}}{X_{4}}+\frac{\mathrm{d} X_{2}}{X_{2}} \cdot \frac{\mathrm{d} X_{4}}{X_{4}}+\frac{\mathrm{d} X_{3}}{X_{3}} \cdot \frac{\mathrm{d} X_{4}}{X_{4}} .
\end{aligned}
$$

From (47), Lemma (3.1), (44), (46), (37), (42),

$$
\begin{aligned}
\frac{\mathrm{d}_{j} \hat{M}}{\hat{M}} & =\frac{\mathrm{d}_{j} A_{j, 1}^{\alpha}}{A_{j, 1}^{\alpha}}+\frac{\mathrm{d}_{j} A_{k, 1}^{\alpha}}{A_{k, 1}^{\alpha}}+\frac{\mathrm{d}_{j} H^{-\frac{c}{6}}}{H^{-\frac{c}{6}}}+\frac{\mathrm{d}_{j} Y}{\mathrm{~d}_{j} Y}+\frac{\mathrm{d}_{j} A_{j, 1}^{\alpha}}{A_{j, 1}^{\alpha}} \cdot \frac{\mathrm{d}_{j} Y}{\mathrm{~d}_{j} Y} \\
& =\alpha \frac{A_{j, 2}}{A_{j, 2}} \mathrm{~d} \xi_{j}\left(t_{j}\right)+\frac{1}{\kappa} \Lambda_{j}\left(X_{j}\right) A_{j, 1} \mathrm{~d} \xi_{j}\left(t_{j}\right)+\left(\frac{1}{6} \alpha A_{j, 1}^{2}-\frac{1}{6} \alpha\right) \mathrm{d} t_{j} .
\end{aligned}
$$

Define $M\left(t_{1}, t_{2}\right),\left(t_{1}, t_{2}\right) \in \mathcal{D}$ :

$$
M\left(t_{1}, t_{2}\right)=\frac{\hat{M}\left(t_{1}, t_{2}\right) \hat{M}(0,0)}{\hat{M}\left(t_{1}, 0\right) \hat{M}\left(0, t_{2}\right)} .
$$

Obviously, $M$ is a positive, and $M(\cdot, 0) \equiv M(0, \cdot) \equiv 1$.

Proposition 4.4. (i) Fix any $\mathcal{F}_{t}^{2}$-stopping time $t_{2} \in\left[0, T_{2}\right)$, $\left(M\left(t_{1}, t_{2}\right), t_{1} \in\left[0, T_{1}\left(t_{2}\right)\right)\right)$ is a $\left(\mathcal{F}_{t_{1}}^{1} \times \mathcal{F}_{t_{2}}^{2}\right)_{t_{1} \geq 0}$-adapted continuous local martingale, and 


$$
\frac{\mathrm{d}_{1} M\left(t_{1}, t_{2}\right)}{M\left(t_{1}, t_{2}\right)}=\left[\left(3-\frac{\kappa}{2}\right) \frac{A_{1,2}}{A_{1,1}}+A_{1,1} \Lambda_{1}\left(X_{1}\right)-\Lambda_{1}\left(\xi_{1}\left(t_{1}\right)-g_{1}\left(t_{1}, a_{2}\right)\right)\right] \frac{\mathrm{d} B_{1}\left(t_{1}\right)}{\sqrt{\kappa}} .
$$

(ii) Fix any $\mathcal{F}_{t}^{1}$-stopping time $t_{1} \in\left[0, T_{1}\right),\left(M\left(t_{1}, t_{2}\right), t_{2} \in\left[0, T_{2}\left(t_{1}\right)\right)\right)$ is a $\left(\mathcal{F}_{t_{1}}^{1} \times \mathcal{F}_{t_{2}}^{2}\right)_{t_{2} \geq 0}$-adapted continuous local martingale, and

$$
\frac{\mathrm{d}_{2} M\left(t_{1}, t_{2}\right)}{M\left(t_{1}, t_{2}\right)}=\left[\left(3-\frac{\kappa}{2}\right) \frac{A_{2,2}}{A_{2,1}}+A_{2,1} \Lambda_{2}\left(X_{2}\right)-\Lambda_{2}\left(\xi_{2}\left(t_{2}\right)-g_{2}\left(t_{2}, a_{1}\right)\right)\right] \frac{\mathrm{d} B_{2}\left(t_{2}\right)}{\sqrt{\kappa}} .
$$

Proof. (i) From Lemma 4.1, we have

$$
\mathrm{d}_{1} \frac{1}{\hat{M}\left(t_{1}, 0\right)}=-\frac{1}{\hat{M}\left(t_{1}, 0\right)^{2}} \mathrm{~d}_{1} \hat{M}\left(t_{1}, 0\right)+\frac{1}{\hat{M}\left(t_{1}, 0\right)^{3}}\left(\mathrm{~d}_{1} \hat{M}\left(t_{1}, 0\right)\right)^{2},
$$

then

$$
\hat{M}\left(t_{1}, 0\right) \mathrm{d}_{1} \frac{1}{\hat{M}\left(t_{1}, 0\right)}=-\frac{1}{\hat{M}\left(t_{1}, 0\right)} \mathrm{d}_{1} \hat{M}\left(t_{1}, 0\right)+\frac{1}{\hat{M}\left(t_{1}, 0\right)^{2}}\left(\mathrm{~d}_{1} \hat{M}\left(t_{1}, 0\right)\right)^{2} .
$$

From (48) and (51) we get

$$
\begin{gathered}
\frac{\mathrm{d}_{1} M\left(t_{1}, t_{2}\right)}{M\left(t_{1}, t_{2}\right)}=\left(\mathrm{d}_{1} \frac{M\left(t_{1}, t_{2}\right)}{M\left(t_{1}, 0\right)}\right) /\left(\frac{\hat{M}\left(t_{1}, t_{2}\right)}{\hat{M}\left(t_{1}, 0\right)}\right) \\
=\frac{\hat{M}\left(t_{1}, 0\right)}{\hat{M}\left(t_{1}, t_{2}\right)}\left(\frac{1}{\hat{M}\left(t_{1}, 0\right)} \mathrm{d}_{1} \hat{M}\left(t_{1}, t_{2}\right)+\hat{M}\left(t_{1}, t_{2}\right) \mathrm{d}_{1} \frac{1}{\hat{M}\left(t_{1}, t_{2}\right)}\right. \\
\left.\quad+\mathrm{d}_{1} \hat{M}\left(t_{1}, t_{2}\right) \cdot \mathrm{d}_{1} \frac{1}{\hat{M}\left(t_{1}, t_{2}\right)}\right) \\
=\frac{\mathrm{d}_{1} \hat{M}\left(t_{1}, t_{2}\right)}{\hat{M}\left(t_{1}, t_{2}\right)}+\hat{M}\left(t_{1}, 0\right) \mathrm{d}_{1} \frac{1}{\hat{M}\left(t_{1}, 0\right)}+\frac{\mathrm{d}_{1} \hat{M}\left(t_{1}, t_{2}\right)}{\hat{M}\left(t_{1}, t_{2}\right)} \cdot\left(\hat{M}\left(t_{1}, 0\right) \mathrm{d}_{1} \frac{1}{\hat{M}\left(t_{1}, 0\right)}\right) \\
=\frac{\mathrm{d}_{1} \hat{M}\left(t_{1}, t_{2}\right)}{\hat{M}\left(t_{1}, t_{2}\right)}-\frac{\mathrm{d}_{1} \hat{M}\left(t_{1}, 0\right)}{\hat{M}\left(t_{1}, 0\right)}+\left(\frac{\mathrm{d}_{1} \hat{M}\left(t_{1}, 0\right)}{\hat{M}\left(t_{1}, 0\right)}\right)^{2}-\left(\frac{\mathrm{d}_{1} \hat{M}\left(t_{1}, t_{2}\right)}{\hat{M}\left(t_{1}, t_{2}\right)}\right) \cdot\left(\frac{\mathrm{d}_{1} \hat{M}\left(t_{1}, 0\right)}{\hat{M}\left(t_{1}, 0\right)}\right) \\
=\left[\left(3-\frac{\kappa}{2}\right) \frac{A_{1,2}}{A_{1,1}}+A_{1,1} \Lambda_{1}\left(X_{1}\right)-\Lambda_{1}\left(\xi_{1}\left(t_{1}\right)-g_{1}\left(t_{1}, a_{2}\right)\right)\right] \frac{\mathrm{d}_{1} B_{1}\left(t_{1}\right)}{\sqrt{\kappa}} .
\end{gathered}
$$

(ii) Similarly,

$\frac{\mathrm{d}_{2} M\left(t_{1}, t_{2}\right)}{M\left(t_{1}, t_{2}\right)}=\left[\left(3-\frac{\kappa}{2}\right) \frac{A_{2,2}}{A_{2,1}}+A_{2,1} \Lambda_{2}\left(X_{2}\right)-\Lambda_{2}\left(\xi_{2}\left(t_{2}\right)-g_{2}\left(t_{2}, a_{1}\right)\right)\right] \frac{\mathrm{d}_{2} B_{2}\left(t_{2}\right)}{\sqrt{\kappa}}$.

Let $\mathcal{J}$ be the set of simple curves between $\mathbb{R}_{\pi}$ and $\mathbb{R}$ with only two endpoint in $\mathbb{R}$, for $J \in \mathcal{J}, i=1,2$, let $T_{j}(J)$ be the first time that $\beta_{j}$ visit $J$. Let $\mathcal{J}^{2}=\left\{\left(J_{1}, J_{2}\right) \mid J_{1}, J_{2} \in \mathcal{J}, J_{1} \cap J_{2}=\varnothing\right.$, $J_{1}$ is on the left side of $\left.J_{2}\right\} \quad$ then $\forall\left(J_{1}, J_{2}\right) \in \mathcal{J}$, when $t_{1} \leq T_{1}\left(J_{1}\right), t_{2} \leq T_{2}\left(J_{2}\right), \quad \beta_{1}\left(\left(0, t_{1}\right]\right) \cap \beta_{2}\left(\left(0, t_{2}\right]\right)=\varnothing$. thus, $\left[0, T_{1}\left(J_{1}\right)\right] \times\left[0, T_{2}\left(J_{2}\right)\right] \subset \mathcal{D}$.

Proposition 4.5. (Boundedness) Fix $J_{1}, J_{2} \in \mathcal{J}^{2}$, then $|\ln (M)|$ is bounded on $\left[0, T_{1}\left(J_{1}\right)\right] \times\left[0, T_{2}\left(J_{2}\right)\right]$ by a constant depend on $J_{1}$ and $J_{2}$ only.

Proof. We say a function is uniformly bounded if the absolute value of func- 
tion is bounded on $\left[0, T_{1}\left(J_{1}\right)\right] \times\left[0, T_{2}\left(J_{2}\right)\right]$ by a constant depend on $J_{1}$ and $J_{2}$ only.

Define

$$
\begin{gathered}
\Gamma_{s_{0}}(x)=\mathrm{e}^{-\frac{s_{0} x}{\kappa}} \Gamma(x), \quad Y_{s_{0}}=\Gamma_{s_{0}}\left(X_{1}\right), \\
\hat{M}_{s_{0}}=A_{1,1}^{\alpha} A_{2,1}^{\alpha} H^{-\frac{c}{6}} Y_{s_{0}}, \quad \hat{M}_{s_{0}}=A_{1,1}^{\alpha} A_{2,1}^{\alpha} H^{-\frac{c}{6}} Y_{s_{0}} .
\end{gathered}
$$

From [10], Lemma $4.4, \ln \left(A_{j, 1}\right), \ln \left(A_{j, 2}\right)$ are uniformly bounded, when $\left(t_{1}, T_{2}\right) \in\left[0, T_{1}\left(J_{1}\right)\right] \times\left[0, T_{2}\left(J_{2}\right)\right]$, exist $m>0$, such that

$$
\begin{gathered}
\left|X_{j}\left(t_{1}, t_{2}\right)\right|=\left|G_{k, t_{k}}\left(t_{j}, \xi_{j}\left(t_{j}\right)\right)-g_{k, t_{k}}\left(t_{j}, \xi_{k}\left(t_{k}\right)\right)\right| \geq m \\
1 /\left(\mathrm{e}^{\frac{x}{2}}-\mathrm{e}^{-\frac{x}{2}}\right)^{2} \text { is a decreasing function, so } 1 /\left(\mathrm{e}^{\frac{x_{j}}{2}}-\mathrm{e}^{-\frac{X_{j}}{2}}\right)^{2} \text { is uniformly }
\end{gathered}
$$

bounded.

$\operatorname{coth}_{2} x$ and $\operatorname{coth}_{2} X_{j}$ are uniformly bounded, so $\operatorname{coth}_{2}^{\prime \prime \prime} X_{j}=\left(1-3 \operatorname{coth}_{2}^{2} X_{j}\right) /\left(\mathrm{e}^{\frac{x_{j}}{2}}-\mathrm{e}^{-\frac{X_{j}}{2}}\right)^{2}$ is uniformly bounded.

From (36) we get, $\ln (H)$ is uniformly bounded on $\left[0, T_{1}\left(J_{1}\right)\right] \times\left[0, T_{2}\left(J_{2}\right)\right]$.

From (5) we have

$$
\Gamma_{s_{0}}(x+2 \pi)=\mathrm{e}^{-\frac{s_{0}(x+2 \pi)}{\kappa}} \Gamma(x+2 \pi)=\mathrm{e}^{-\frac{s_{0}(x+2 \pi)}{\kappa}} \mathrm{e}^{\frac{2 \pi s_{0}}{\kappa}} \Gamma(x)=\mathrm{e}^{\frac{s_{0} x}{\kappa}} \Gamma(x)=\Gamma_{s_{0}}(x),
$$

so $\Gamma_{s_{0}}$ is a continuous function with period $2 \pi$. Then $Y_{s_{0}}$ is uniformly bounded on $\left[0, T_{1}\left(J_{1}\right)\right] \times\left[0, T_{2}\left(J_{2}\right)\right]$. Thus, $\ln \hat{M}_{s_{0}}$ is uniformly bounded on $\left[0, T_{1}\left(J_{1}\right)\right] \times\left[0, T_{2}\left(J_{2}\right)\right]$.

Since

$$
\ln M\left(t_{1}, t_{2}\right)=\left(\ln M\left(t_{1}, t_{2}\right)-\ln M_{s_{0}}\left(t_{1}, t_{2}\right)\right)+\ln M_{s_{0}}\left(t_{1}, t_{2}\right),
$$

It is suffices to proof that $\ln M\left(t_{1}, t_{2}\right)-\ln M_{s_{0}}\left(t_{1}, t_{2}\right)$ is uniformly bounded on $\left[0, T_{1}\left(J_{1}\right)\right] \times\left[0, T_{2}\left(J_{2}\right)\right]$.

From (49), (47) we have,

$$
\begin{aligned}
& \ln \left(M\left(t_{1}, t_{2}\right)\right)-\ln \left(M_{s_{0}}\left(t_{1}, t_{2}\right)\right) \\
&=\left(\ln \left(\hat{M}\left(t_{1}, t_{2}\right)\right)-\ln \left(\hat{M}_{s_{0}}\left(t_{1}, t_{2}\right)\right)\right)+\left(\ln (\hat{M}(0,0))-\ln \left(\hat{M}_{s_{0}}(0,0)\right)\right) \\
&-\left(\ln \left(\hat{M}\left(t_{1}, 0\right)\right)-\ln \left(\hat{M}_{s_{0}}\left(t_{1}, 0\right)\right)\right)-\left(\ln \left(\hat{M}\left(0, t_{2}\right)\right)-\ln \left(\hat{M}_{s_{0}}\left(0, t_{2}\right)\right)\right) \\
&=\left(\ln Y\left(t_{1}, t_{2}\right)-\ln Y_{s_{0}}\left(t_{1}, t_{2}\right)\right)+\left(\ln Y(0,0)-\ln Y_{s_{0}}(0,0)\right) \\
&-\left(\ln Y\left(t_{1}, 0\right)-\ln Y_{s_{0}}\left(t_{1}, 0\right)\right)-\left(\ln Y\left(0, t_{2}\right)-\ln Y_{s_{0}}\left(0, t_{2}\right)\right) \\
&= \frac{s_{0}}{\kappa}\left(X_{1}\left(t_{1}, t_{2}\right)+X_{1}(0,0)-X_{1}\left(t_{1}, 0\right)-X_{1}\left(0, t_{2}\right)\right) .
\end{aligned}
$$

Let $G\left(t_{1}, t_{2}\right)=G_{2, t_{2}}\left(t_{1}, \xi_{1}\left(t_{1}\right)\right), g\left(t_{1}, t_{2}\right)=g_{1, t_{2}}\left(t_{1}, \xi_{2}\left(t_{2}\right)\right)$. From (23) we see that $X_{1}=G-g$. It is suffices to proof that $G\left(t_{1}, t_{2}\right)-G\left(t_{1}, 0\right)-G\left(0, t_{2}\right)+G(0,0)$ 
and $g\left(t_{1}, t_{2}\right)-g\left(t_{1}, 0\right)-g\left(0, t_{2}\right)+g(0,0)$ are uniformly bounded. From (31) we have

$$
\begin{aligned}
g\left(t_{1}, t_{2}\right)-g\left(0, t_{2}\right) & =\int_{0}^{t_{1}} \partial_{s} g_{1, t_{2}}\left(s, \xi_{2}\left(t_{2}\right)\right) \mathrm{d} s \\
& =-\int_{0}^{t_{1}} A_{j, 1}^{2}\left(s_{1}, t_{2}\right) \operatorname{coth}_{2}\left(X_{j}\left(s, t_{2}\right)\right) \mathrm{d} s .
\end{aligned}
$$

$A_{j, 1}^{2}$ and $\operatorname{coth}_{2} X_{j}$ are uniformly bounded, so $g\left(t_{1}, t_{2}\right)-g\left(0, t_{2}\right)$ is uniformly bounded.

So $g\left(t_{1}, t_{2}\right)-g\left(t_{1}, 0\right)-g\left(0, t_{2}\right)+g(0,0)$ is uniformly bounded.

Let $\hat{G}\left(t_{1}, t_{2}\right)=G\left(t_{1}, t_{2}\right)-\xi_{1}\left(t_{1}\right)$, then

$$
\begin{aligned}
& G\left(t_{1}, t_{2}\right)-G\left(t_{1}, 0\right)-G\left(0, t_{2}\right)-G(0,0) \\
& =\hat{G}\left(t_{1}, t_{2}\right)-\hat{G}\left(t_{1}, 0\right)-\hat{G}\left(0, t_{2}\right)+\hat{G}(0,0) .
\end{aligned}
$$

It is suffices to proof that $\hat{G}\left(t_{1}, t_{2}\right)$ is uniformly bounded. In fact,

$$
\begin{aligned}
& \left|\hat{G}\left(t_{1}, t_{2}\right)\right|=\left|G\left(t_{1}, t_{2}\right)-\xi_{1}\left(t_{1}\right)\right| \\
= & \left|\left(g_{1, t_{2}}\left(t_{1}, \cdot\right) \circ g_{2}\left(t_{2}, \cdot\right) \circ g_{1}^{-1}\left(t_{1}, \xi_{1}\left(t_{1}\right)\right)\right)-\xi_{1}\left(t_{1}\right)\right| \\
= & \mid\left(g_{1, t_{2}}\left(t_{1}, \cdot\right) \circ g_{2}\left(t_{2}, \cdot\right) \circ g_{1}^{-1}\left(t_{1}, \xi_{1}\left(t_{1}\right)\right)-g_{2}\left(t_{2}, \cdot\right) \circ g_{1}^{-1}\left(t_{1}, \xi_{1}\left(t_{1}\right)\right)\right) \\
& +\left(g_{2}\left(t_{2}, \cdot\right) \circ g_{1}^{-1}\left(t_{1}, \xi_{1}\left(t_{1}\right)\right)-g_{1}^{-1}\left(t_{1}, \xi_{1}\left(t_{1}\right)\right)\right)+\left(g_{1}^{-1}\left(t_{1}, \xi_{1}\left(t_{1}\right)\right)-\xi_{1}\left(t_{1}\right)\right) \mid \\
\leq & \left|g_{1, t_{2}}\left(t_{1}, \cdot\right) \circ g_{2}\left(t_{2}, \cdot\right) \circ g_{1}^{-1}\left(t_{1}, \xi_{1}\left(t_{1}\right)\right)\right|+\left|g_{2}\left(t_{2}, \cdot\right) \circ g_{1}^{-1}\left(t_{1}, \xi_{1}\left(t_{1}\right)\right)\right| \\
& +\left|\left(g_{2}\left(t_{2}, \cdot\right) \circ g_{1}^{-1}\left(t_{1}, \xi_{1}\left(t_{1}\right)\right)-g_{1}^{-1}\left(t_{1}, \xi_{1}\left(t_{1}\right)\right)\right)+\left(g_{1}^{-1}\left(t_{1}, \xi_{1}\left(t_{1}\right)\right)-\xi_{1}\left(t_{1}\right)\right)\right| .
\end{aligned}
$$

Similarly, we can prove that the other parts of the formula above are uniformly bounded. Thus, $\hat{G}\left(t_{1}, t_{2}\right)$ is uniformly bounded is proved.

\subsection{Coupling Measure}

Let $\mu_{j}$ denote the distribution of $\left(\xi_{j}\right), j=1,2$. Let $\mu=\mu_{1} \times \mu_{2} . \xi_{1}$ and $\xi_{2}$ be independent, so $\mu$ is the joint distribution of $\left(\xi_{1}\right)$ and $\left(\xi_{2}\right)$. Fix $\left(J_{1}, J_{2}\right) \in \mathcal{J}^{2}$, from the properties of local martingale and proposition 4.1, $E_{\mu}\left[M\left(T_{1}\left(J_{1}\right), T_{2}\left(J_{2}\right)\right)\right]=M(0,0)=1$.

Define $v_{J_{1}, J_{2}}$ by $\mathrm{d} v_{J_{1}, J_{2}} / \mathrm{d} \mu=M\left(T_{1}\left(J_{1}\right), T_{2}\left(J_{2}\right)\right)$, then $v_{J_{1}, J_{2}}$ is a probability measure. Let $v_{1}$ and $v_{2}$ are marginal measure of $v_{J_{1}, J_{2}}$.

$$
\frac{\mathrm{d} v_{1}}{\mathrm{~d} \mu_{1}}=M\left(T_{1}\left(J_{1}\right), 0\right)=1, \quad \frac{\mathrm{d} v_{2}}{\mathrm{~d} \mu_{2}}=M\left(T_{2}\left(J_{2}\right), 0\right)=1 .
$$

So, $v_{j}=\mu_{j}, j=1,2$. Suppose $\left(\xi_{1}\right)$ and $\left(\xi_{2}\right)$ are the joint distribution of $v_{J_{1}, J_{2}}$. For each $\left(\xi_{j}\right)$, we have the joint distribution of $\left(\xi_{j}\right)$ is $\mu_{j}$.

The proof of Theorem 4.1: Fix an $\left(\mathcal{F}_{t}^{2}\right)$-stopping time $t_{2} \leq T_{2}\left(J_{2}\right)$. From (39), (50) and Girsnov theorem. Under the measure of $v_{J_{1}, J_{2}}$, exist an $\left(\mathcal{F}_{t_{1}}^{1} \times \mathcal{F}_{t_{2}}^{2}\right)_{t_{1} \geq 0}$-Brownian motion $\hat{B}_{1, t_{2}}\left(t_{1}\right)$, such that $\xi_{1}\left(t_{1}\right), 0 \leq t_{1} \leq T_{1}\left(J_{1}\right)$ satisfy $\left(\mathcal{F}_{t_{1}}^{1} \times \mathcal{F}_{t_{2}}^{2}\right)$-adapted SDE:

$$
\begin{aligned}
\mathrm{d} \xi_{1}\left(t_{1}\right)= & \sqrt{\kappa} A_{1,1} \mathrm{~d} \hat{B}_{1, t_{2}}\left(t_{1}\right)+A_{1,1} \Lambda_{1}\left(\xi_{1, t_{2}}\left(t_{1}\right)-g_{1, t_{2}}\left(t_{1}, \xi_{2}\left(t_{2}\right)\right)\right) \mathrm{d} t_{1} \\
& -\left(\frac{\kappa}{2}-3\right) \frac{A_{1,2}}{A_{1,1}} \mathrm{~d} t_{1} .
\end{aligned}
$$


From the formula above and (6), (14) and Itô formula,

$$
\begin{aligned}
\mathrm{d} \xi_{1, t_{2}}\left(t_{1}\right) & =\mathrm{d} G_{2, t_{2}}\left(t_{1}, \xi_{1}\left(t_{1}\right)\right) \\
& =A_{1,1} \mathrm{~d} \xi_{1}\left(t_{1}\right)+\left(\frac{\kappa}{2}-3\right) A_{1,2} \mathrm{~d} t_{1} \\
& =\sqrt{\kappa} A_{1,1} \mathrm{~d} \hat{B}_{1, t_{2}}\left(t_{1}\right)+A_{1,1}^{2} \Lambda_{1} \xi_{1, t_{2}}\left(t_{1}\right)-g_{1, t_{2}}\left(t_{1}, \xi_{2}\left(t_{2}\right)\right) \mathrm{d} t_{1} .
\end{aligned}
$$

Since $\eta_{1, t_{2}}\left(t_{1}\right)=\xi_{1, t_{2}}\left(u_{1, t_{2}}^{-1}\left(t_{1}\right)\right), f_{1, t_{2}}\left(t_{1}, \cdot\right)=g_{1, t_{2}}\left(u_{1, t_{2}}^{-1}\left(t_{1}\right), \cdot\right)$, from $(8)$, there is a Brownian motion $\hat{B}_{1, t_{2}}\left(s_{1}\right)$ such that for $0 \leq s_{1} \leq u_{1, t_{2}}\left(T_{1}\left(s_{1}\right)\right)$,

$$
\begin{gathered}
\mathrm{d} \eta_{1, t_{2}}\left(t_{1}\right)=\sqrt{\kappa} \mathrm{d} \hat{B}_{1, t_{2}}\left(t_{1}\right)+\Lambda_{1}\left(\eta_{1, t_{2}}\left(t_{1}\right)-f_{1, t_{2}}\left(t_{1}, \xi_{2}\left(t_{2}\right)\right)\right) \mathrm{d} t_{1}, \\
\eta_{1, t_{2}}(0)=\xi_{1, t_{2}}\left(t_{1}\right)=G_{2, t_{2}}\left(0, \xi_{1}(0)\right)=g_{2}\left(t_{2}, a_{1}\right) .
\end{gathered}
$$

Thus, after a time-change, $g_{2}\left(t_{2}, \gamma_{1}\left(t_{1}\right)\right), 0 \leq t_{1} \leq T_{1}\left(J_{1}\right)$, is a strip a $\operatorname{SLE}(\kappa, \Lambda)$ trace in $\mathbb{S}_{\pi}$ started from $g_{2}\left(t_{2}, a_{1}\right)$ with marked point $\xi_{2}\left(t_{2}\right)$. This shows that, conditioning on $\mathcal{F}_{t_{2}}^{2}$, after a time-change, $\gamma_{1}\left(t_{1}\right), 0 \leq t_{1} \leq T_{1}\left(J_{1}\right)$ is a strip $\operatorname{SLE}(\kappa, \Lambda)$ trace in $\mathbb{S}_{\pi} \backslash \beta_{2}\left(t_{2}\right)$ started from $a_{1}$ with marked point $\gamma_{2}\left(t_{2}\right)$.

\section{Conclusion}

In this paper, $A$ bounded continuous local martingale $M$ based on ordinary differential Equation ((4), (5)) is constructed. On this basis, we prove that for $\kappa \in(0,4]$, there is a coupling of two strip $\mathrm{SLE}_{\kappa}$ traces on the strip domain. The method in this article can provide reference for the study of stochastic coupling of SLE on disk and other regions. The conclusion of this paper can be used to study the reversibility of SLE on the strip domain.

\section{Conflicts of Interest}

The author declares no conflicts of interest regarding the publication of this paper.

\section{References}

[1] Schramm, O. (2000) Scaling Limits of Loop-Erased Random Walks and Uniform Spanning Trees. Israel Journal of Mathematics, 118, 221-288. https://doi.org/10.1007/BF02803524

[2] Smirnov, S. (2001) Critical Percolation in the Plane: Conformal Invariance, Cardy's Formula, Scaling Limits. Comptes Rendus de P Académie des Sciences, 333, 239-244. https://doi.org/10.1016/S0764-4442(01)01991-7

[3] Lawler, G.F., Schramm, O. and Werner, W. (2001) The Dimension of the Planar Brownian Frontier Is 4/3. Mathematical Research Letters, 8, 401-411. https://doi.org/10.4310/MRL.2001.v8.n4.a1

[4] Lawler, G.F., Schramm, O. and Werner, W. (2004) Conformal Invariance of Planar Loop-Erased Random Walks and Uniform Spanning Trees. Annals of Probability, 32, 939-995. https://doi.org/10.1214/aop/1079021469

[5] Schramm, O. and Sheffield, S. (2005) Harmonic Explorer and Its Convergence to SLE4. Annals of Probability, 33, 2127-2148. https://doi.org/10.1214/009117905000000477 
[6] Cardy, J. (2005) SLE for Theoretical Physicists. Annals of Physics, 318, 81-118. https://doi.org/10.1016/j.aop.2005.04.001

[7] Schramm, O. and Sheffield, S. (2009) Contour Lines of the Two-Dimensional Discrete Gaussian Free Field. Acta Mathematica, 202, 21-137.

https://doi.org/10.1007/s11511-009-0034-y

[8] Smirnov, S. (2010) Conformal Invariance in Random Cluster Models. I. Holmorphic Fermions in the Ising Model. Annals of Mathematics, 172, 1435-1467. https://doi.org/10.4007/annals.2010.172.1435

[9] Zhan, D. (2008) Reversibility of Chordal SLE. Annals of Probability, 36, 1472-1494. https://doi.org/10.1214/07-AOP366

[10] Zhan, D. (2015) Reversibility of Whole-Plane SLE. Probability Theory and Related Fields, 161, 561-618. https://doi.org/10.1007/s00440-014-0554-Z

[11] Dubédat, J. (2005) SLE $(\kappa, \rho)$ Martingales and Duality. Annals of Probability, 33, 223-243. https://doi.org/10.1214/009117904000000793

[12] Dubèdat, J. (2007) Commutation Relations for Schramm-Loewner Evolutions. Communications on Pure and Applied Mathematics, 60, 1792-1847. https://doi.org/10.1002/cpa.20191

[13] Bauer, M., Bernard, D. and Houdayer, J. (2005) Dipolar Stochastic Loewner Evolutions. Journal of Statistical Mechanics. Theory and Experiment, No. 3, P03001. https://doi.org/10.1088/1742-5468/2005/03/P03001

[14] Zhan, D. (2004) Random Loewner Chains in Riemann Surfaces. Ph.D. Thesis, Caltech.

[15] Zhan, D. (2012) Restriction Properties of Annulus SLE. Journal of Statistical Physics, 146, 1026-1058. https://doi.org/10.1007/s10955-012-0438-5 